CINÉTIQUE ENZYMATIQUE 


\section{Grenoble Sciences}

Grenoble Sciences poursuit un triple objectif :

- réaliser des ouvrages correspondant à un projet clairement défini, sans contrainte de mode ou de programme,

- garantir les qualités scientifique et pédagogique des ouvrages retenus,

- proposer des ouvrages à un prix accessible au public le plus large possible.

Chaque projet est sélectionné au niveau de Grenoble Sciences avec le concours de referees anonymes. Puis les auteurs travaillent pendant une année (en moyenne) avec les membres d'un comité de lecture interactif, dont les noms apparaissent au début de l'ouvrage. Celui-ci est ensuite publié chez l'éditeur le plus adapté.

(Contact : Tél. : (33)4 76514695 - E-mail : Grenoble.Sciences@ujf-grenoble.fr)

Deux collections existent chez EDP Sciences :

- la Collection Grenoble Sciences, connue pour son originalité de projets et sa qualité

- Grenoble Sciences - Rencontres Scientifiques, collection présentant des thèmes de recherche d'actualité, traités par des scientifiques de premier plan issus de disciplines différentes.

\section{Directeur scientifique de Grenoble Sciences}

Jean BorNAREL, Professeur à l'Université Joseph Fourier, Grenoble 1

\section{Comité de lecture pour Cinétique enzymatique}

- Serge Chesne, Maître de Conférences à l'Université de La Réunion

- Jean-Marie FrÈre, Professeur à l'Université de Liège

- Michel VAN DER REST, Professeur à l'Ecole Nationale Supérieure de Lyon, Directeur-adjoint de la Recherche

et

- Julien BRÉVIER

Grenoble Sciences reçoit le soutien du Ministère de l'Éducation nationale, du Ministère de la Recherche, et de la Région Rhône-Alpes.

Réalisation et mise en pages : Centre technique Grenoble Sciences

Illustration de couverture : Alice GIRAUD

à partir d'une image originale représentant la structure de la créatine kinase fournie par le Professeur Theo Wallimann et le Docteur Uwe SchlatTNER de ETH de Zurich (Suisse)

ISBN 2-86883-742-5

(C) EDP Sciences, 2005

CPortland Press, Ltd. London, 2004

This translation of portions of FUNDAMENTALS OF ENZYME KINETICS first published in $(1995,2004)$ is published by arrangement with Portland Press, London 


\section{CINÉTIQUE ENZYMATIQUE}

Athel CORNISH-BOWDEN

Marc JAMIN

Valdur SAKS 


\section{Ouvrages Grenoble Sciences édités par EDP Sciences}

\section{Collection Grenoble Sciences}

Chimie. Le minimum à savoir (J. Le Coarer) - Electrochimie des solides (C. Déportes et al.) - Thermodynamique chimique (M. Oturan \& M. Robert) - Chimie organométallique (D. Astruc) - De l'atome à la réaction chimique (sous la direction de R. Barlet)

Introduction à la mécanique statistique (E. Belorizky \& W. Gorecki) - Mécanique statistique. Exercices et problèmes corrigés (E. Belorizky \& W. Gorecki) - La cavitation. Mécanismes physiques et aspects industriels (J.P. Franc et al.) - La turbulence (M. Lesieur) - Magnétisme : I Fondements, II Matériaux et applications (sous la direction d'E. du Trémolet de Lacheisserie) - Du Soleil à la Terre. Aéronomie et météorologie de l'espace (J. Lilensten \& P.L. Blelly) - Sous les feux du Soleil. Vers une météorologie de l'espace (J. Lilensten \& $J$. Bornarel) - Mécanique. De la formulation lagrangienne au chaos hamiltonien (C. Gignoux $\&$ B. Silvestre-Brac) - Problèmes corrigés de mécanique et résumés de cours. De Lagrange à Hamilton $(C$. Gignoux \& B. Silvestre-Brac) - La mécanique quantique. Problèmes résolus, T. 1 et 2 (V.M. Galitsky, B.M. Karnakov \& V.I. Kogan) - Analyse statistique des données expérimentales (K. Protassov) - Description de la symétrie. Des groupes de symétrie aux structures fractales (J. Sivardière) - Symétrie et propriétés physiques. Du principe de Curie aux brisures de symétrie (J. Sivardière)

Exercices corrigés d'analyse, T. 1 et 2 (D. Alibert) - Introduction aux variétés différentielles (J. Lafontaine) - Analyse numérique et équations différentielles (J.P. Demailly) - Mathématiques pour les sciences de la vie, de la nature et de la santé (F.\& J.P. Bertrandias) - Approximation hilbertienne. Splines, ondelettes, fractales (M. Attéia \& J. Gaches) - Mathématiques pour l'étudiant scientifique, T. 1 et 2 (Ph.J. Haug)

Bactéries et environnement. Adaptations physiologiques (J. Pelmont) - Enzymes. Catalyseurs du monde vivant (J. Pelmont) - La plongée sous-marine à l'air. L'adaptation de l'organisme et ses limites (Ph. Foster) - Endocrinologie et communications cellulaires (S. Idelman \& $J$. Verdetti) - Eléments de biologie à l'usage d'autres disciplines (P. Tracqui \& J. Demongeot Bioénergétique (B. Guérin)

L'Asie, source de sciences et de techniques (M. Soutif) - La biologie, des origines à nos jours (P. Vignais) - Naissance de la physique. De la Sicile à la Chine (M. Soutif) - Le régime oméga 3. Le programme alimentaire pour sauver notre santé (A. Simopoulos, J. Robinson, M. de Lorgeril \& P. Salen) - Gestes et mouvements justes. Guide de l'ergomotricité pour tous (M. Gendrier)

Listening Comprehension for Scientific English (J. Upjohn) - Speaking Skills in Scientific English (J. Upjohn, M.H. Fries \& D. Amadis) - Minimum Competence in Scientific English (S. Blattes, V. Jans \& J. Upjohn)

\section{Grenoble Sciences - Rencontres Scientifiques}

Radiopharmaceutiques. Chimie des radiotraceurs et applications biologiques (sous la direction de M. Comet \& M. Vidal) - Turbulence et déterminisme (sous la direction de M. Lesieur) Méthodes et techniques de la chimie organique (sous la direction de D. Astruc) - L'énergie de demain. Techniques, environnement et économie (sous la direction de H. Nifenecker) 